\title{
DESARROLLO DE UN SCANNER 3D
}

V. Toranzos ${ }^{1}$, L. Zini ${ }^{2}$, A. Busso ${ }^{2}$

\section{RESUMEN}

En el presente trabajo se muestra un primer prototipo de un escáner 3D detallando su construcción mecánica, los circuitos electrónicos que lo componen y la programación asociada a su funcionamiento. Para el mismo se utilizaron componentes de fácil adquisición y programas de desarrolló del tipo libre. Como resultado se llegó a un modelo tridimensional numérico de objetos físicos pudiéndose recomponer este objeto mediante una impresora 3D, mostrando de esta forma su utilidad y precisión alcanzada.

Palabras claves: holografía, imagen 3D, procesamiento de imágenes, Scanner 3D.

\section{INTRODUCCIÓN}

Un escáner 3D es un dispositivo que analiza un objeto o una escena para reunir datos de su forma y ocasionalmente su color (Malik 2011). Un modelo tridimensional digital es una representación numérica de las características visuales del objeto. A partir del modelo digital, es posible calcular una representación realista del objeto en una imagen bidimensional. Esta imagen, mediante el uso de algunas técnicas como prospectivo y el sombreado, puede emular la percepción del ojo humano, dando una representación realista de la tridimensionali- dad del objeto. Un sistema de visualización en $3 \mathrm{D}$, por lo general, está compuesto de dos elementos: La escena, una representación matemática de los objetos tridimensionales, y el procesamiento, la técnica de calcular las imágenes en 2D de la escena.

Las aplicaciones basadas en el procesamiento del modelo tridimensional son hoy muy difundidas gracias a la mayor disponibilidad de dispositivos gráficos tridimensionales y la tendencia a la disminución del costo de la potencia de cálculo. Estas aplicaciones se utilizan en muchos campos como el diseño, la arqueología, la medicina y el entretenimiento. La posibilidad de utilizar el modelo en 3D digital puede tener muchas ventajas. Es posible utilizar el modelo para la simulación digital o para crear una versión digital modificada del objeto. En el campo del entretenimiento, el modelado $3 \mathrm{D}$ permite utilizar objetos reales o personas para la creación de personajes y entornos en la animación digital. Además, para el estudio de grandes objetos como edificios o regiones geográficas que puede ser muy útil el manejo de modelos digitales escalables.

El modelo 3D digital puede venir de dos maneras diferentes: diseño asistido por ordenador (CAD) y medición física del objeto. En un entorno CAD, objetos simples pueden ser representados a través de ecua- 
ciones simples: por ejemplo, la ecuación $\mathrm{x} 2$ $+\mathrm{y} 2+\mathrm{z} 2=\mathrm{R} 2$, puede ser utilizado para la representación de una esfera con radio $\mathrm{R}$. Aunque estas sencillas ecuaciones pueden parecer limitativo, el conjunto de objetos representables se puede ampliar a través de una técnica llamada geometría sólida constructiva (CSG); esta técnica se basa en la combinación de objetos sólidos simples (por ejemplo, cubo, cono, esfera) con el fin de crear objetos más complejos a través de operaciones como unión, intersección, diferencia (por ejemplo, un tubo puede ser visto como una diferencia de dos cilindros con diferentes radios). De todos modos, este método no es adecuado para describir cierta clase de objetos reales y por ello no se utiliza comúnmente. Hoy en día, el software CAD permite la creación de modelos muy complejos que son, en general, basados en Non-Uniform Rational B-Spline (NURBS) (Piegl 1987), un modelo matemático que permite la generación de curvas y superficies con gran flexibilidad y de precisión. E1 NURBS es adecuado para el manejo de ambas formas de análisis y de forma libre.

Por otro lado, la digitalización través de la medición del objeto físico es un proceso que permite obtener el modelo $3 \mathrm{D}$ de una manera semi-automática (Teutsch 2007). Se basa en la medición de características geométricas del objeto y en sus características visuales como el color y la textura. Con respecto a la $\mathrm{CAD}$, la digitalización se caracteriza por un proceso de creación generalmente más rápido y un nivel de precisión más alto (o, al menos, medible). Además, la digitalización, que es sustancialmente un proceso de medición, no requiere habilidades artísticas para el operador.

Muchas veces, la reproducción tridimensional de un objeto, en términos de modelo matemático, es algo complejo de formular por lo que resulta más sencillo simplemente retratarlo, mediante técnicas apropiadas, a fin de que la fotografía obtenida reproduzca sus características de dimensiones espaciales.

\section{MATERIALES Y METODOS}

De manera general podemos decir que la metodología que utilizamos en la obtención de una imagen 3D consiste en una plataforma giratoria, un LASER que recorre verticalmente el objeto y una cámara que toma la escena. El sistema de coordenadas apropiado para esta disposición es el de cilíndricas $(r, \phi, z)$ donde el contorno del objeto nos brinda información sobre el radio " $r$ " de cada punto como se esquematiza en la figura 1, la altura " $z$ " se determina directamente con la cámara y el ángulo " $\phi$ " está dado por la posición de la plataforma.

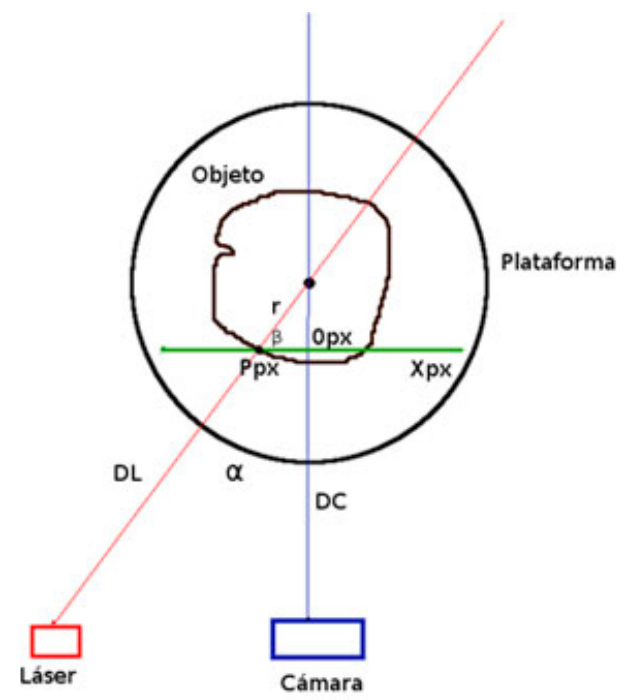

Figura 1.- Diagrama geométrico utilizado para la determinación del radio r de cada punto del objeto.

Para explicar el prototipo de escáner 3D desarrollado dividiremos a este en tres partes funcionales fácilmente distinguibles y que llamaremos: 
1. Montaje mecánico.

2. Circuito electrónico de control.

3. Programa de adquisición y procesamiento de imágenes.

\section{1.- Montaje mecánico:}

El prototipo se ensambló sobre una base de aglomerado de alta densidad (MDF), ubicándose en ella un motor paso a paso cuyo eje se vincula directamente a una plataforma giratoria de $12 \mathrm{~cm}$ de diámetro. Bajo esta plataforma se encuentra un sensor de posición que permite determinar el inicio del escaneo. A $20 \mathrm{~cm}$ del centro de la plataforma giratoria se ubicaron una cámara y un LASER, este último genera una línea vertical perpendicular a la plataforma. La dirección de visual de la cámara guarda un ángulo $\alpha$ respecto a la dirección del LASER, convergiendo estas direcciones en el centro de la plataforma. En la figura 2 se muestra esta disposición indicando sus componentes principales.

\section{2.- Circuito electrónico de control:}

Este componente del escáner es el encargado de controlar el motor de la plataforma y de ubicarla según un sensor óptico que establece la posición inicial de escaneo. Por otro lado permite el encendido y regulación del LASER. Se comunica mediante protocolo RS232 con un adaptador USB y de allí a una computadora personal (PC) donde se adquirirá la imagen, esta disposición se esquematiza en la parte derecha de la figura 2.

El corazón de este circuito, que se muestra completo en la figura 3, es un microcontrolador $(\mu \mathrm{C})$ PIC16F88 (hoja técnica de

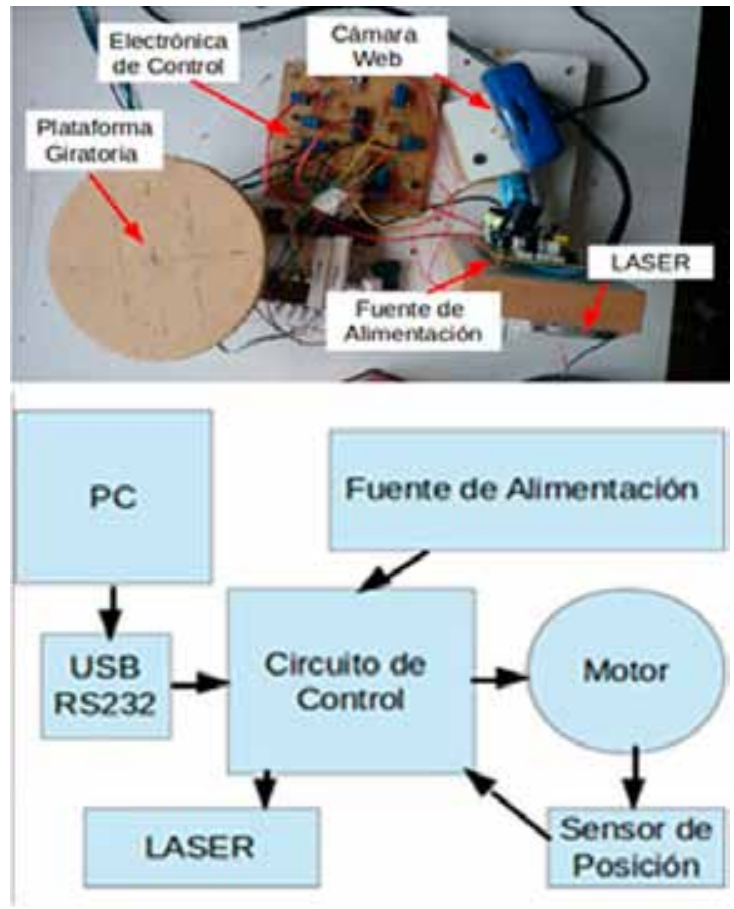

Figura 2.- Arriba: Fotografía desde arriba del prototipo de escáner 3D. Abajo: Descripción esquemática del escáner $3 D$.

Microchip), que tiene incorporado internamente el protocolo de comunicación RS232 permitiendo así la operación de dos puertos entrada/salida de 8 bits (puerto A y puerto B). Las principales funciones del circuito que se muestra en la figura 3 se implementaron de la siguiente manera: 


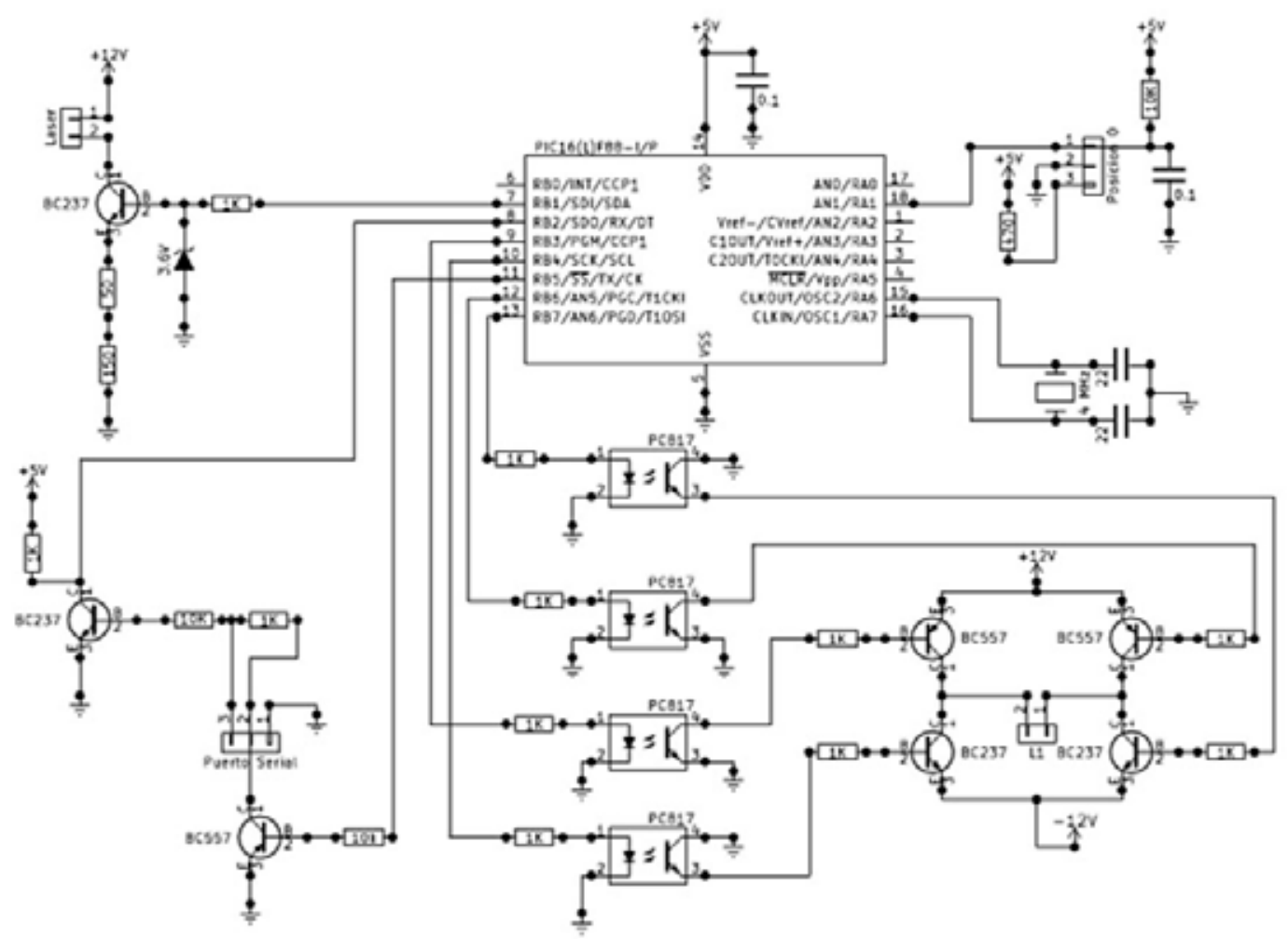

Figura 3.- Diagrama del circuito electrónico de la placa de control realizado en el programa de uso libre Kicad (link de internet Kicad).

Comunicación: Se requiere una adaptación de los niveles de tensión propios del puerto RS232 (+/- $10 \mathrm{~V})$ a los permitidos por el microcontrolador $(0$ y $5 \mathrm{~V})$. Esta función se realizó de manera discreta utilizando dos transistores de uso general. Los puertos utilizados del $\mu \mathrm{C}$ son $\mathrm{B} 2$ y B5.

Control del motor: E1 motor utilizado es del tipo bipolar (Marder 2008), para accionarlo se utilizó un semipuente conformado por un par complementario de transistores bipolares (Millman 1976), requiriéndose dos semipuentres, uno por cada bobina del motor, y una fuente partida de $+/-12 \mathrm{~V}$. La excitación de los transistores se realizó mediante optoacopladores del tipo LEDTransistor PC817 (hoja técnica de Sharp), de modo que ambos transistores del semipuente trabajan con emisor a masa en las fuentes de tensión $-12 \mathrm{Vy}+12 \mathrm{~V}$ respectivamente asegurando su comportamiento en la región de saturación y al mismo tiempo independizando de la fuente de $5 \mathrm{~V}$ con la que se alimenta el uC. Se destinaron a esta función los puertos B3, B4, B6 y B7 del $\mu \mathrm{C}$.

Sensor de posición: Se utilizó un optoacoplador abierto que requiere pocos componentes externos. $\mathrm{E} 1 \mu \mathrm{C}$ obtiene esta señal por el puerto A1.

Control del LASER: Consiste en una fuente de corriente constante de $15 \mathrm{~mA}$ implementada con un transistor bipolar y comandada por el $\mu \mathrm{C}$ mediante el puerto B1.

$\mathrm{E} 1 \mu \mathrm{C}$ fue programado con una rutina 
que atiende constantemente al puerto de comunicación, cuando este recibe un carácter ASCII determinado realiza la acción programada y regresa al modo "escucha" comportándose de esta manera como un esclavo del programa de adquisición que corre en la PC.

Las principales instrucciones son:

$>$ Mover el motor a posición inicial. $\mathrm{Ca}$ rácter "f".

> Mover motor $\mathrm{N}$ pasos en sentido izquierdo "abN" o derecho "aaN".

> Encender “d” o apagar "e" el LASER.

\section{3.- Programa de adquisición y procesa- miento de imágenes:}

Se desarrolló un programa en el lenguaje e programación Python (link de internet Python) que controla la plataforma, obtiene las fotografías y finalmente reconstruye a partir de las imágenes una nube de puntos en 3D correspondiente al objeto escaneado. La elección de Python se debe a su potencia, disponibilidad de un módulo de procesamiento de imágenes y facilidad de programación, además de que es multiplataforma y de libre uso. En esta primera versión se utilizan los siguientes paquetes: Serial, OpenCV, Math, Numpy y PIL. Siguiendo al diseño del programa del microcontrolador, se envían letras para ejecutar cada comando.

Control de la Plataforma: La PC reconoce a la plataforma como un puerto serial, en este caso "ttyUSB0". El programa realiza las siguientes tareas en orden:

$>$ Establece la conexión con la platafor- ma y con la cámara web.

$>$ Coloca la base giratoria en su posición de inicio.

> Prende el LASER.

> Toma fotografías y hacer avanzar un paso al motor. Repetir esto hasta completar los 360 grados.

> Apaga el LASER, retorna a la posición de inicio y libera el motor.

Obtención de Imágenes: La librería OpenCV (link de internet OpenCV) permite interactuar con la cámara web. En primer lugar, se debió realizar una calibración de la misma, para ello se utilizaron las funciones ya incluidas dentro de OpenCV (iink de internet calibración de la cámara).

Una vez calibrada la cámara, el programa se ocupa de sacar una foto por cada paso del motor. Un problema encontrado en la cámara web de bajo costo utilizada es el tiempo que tarda el sensor en ponerse en régimen para lograr fotografías adecuadas. A fin de evitar este inconveniente, el programa obtiene varias fotografías que luego serán descartadas para la reconstrucción final.

Todas las fotografías se almacenan dentro de una carpeta accesible al usuario, por lo que este puede controlar que el proceso se lleve a cabo en forma correcta. Al finalizar, se cuenta con un número de fotografías igual a la cantidad de pasos del motor.

Reconstrucción del Objeto: El siguiente paso del programa es reconstruir una nube de puntos partiendo de las fotos obtenidas anteriormente. El archivo de salida es del 
tipo OBJ (Wavefront Technologies) y en su interior contiene la reconstrucción del objeto en un espacio tridimensional. El algoritmo es el siguiente:

$>$ Primero se selecciona una imagen del directorio creado anteriormente.

$>$ En la imagen está la información correspondiente al ángulo “ $\phi$ ” en el que se encontraba el objeto.

$>$ Se eliminan todos los canales de color, dejando solamente el rojo (mismo color del LASER).

$>$ Se recorre la imagen, para cada pixel de altura " $h$ ", se obtiene la coordenada "px" del pixel de máximo valor.

$>$ Se llama a una sub-función que transforma la coordenada "px" para la altura " $h$ " y el ángulo " $\phi$ ” en un punto de coordenadas espaciales $(\mathrm{x}, \mathrm{y}, \mathrm{z})$.

> Se repite el paso anterior para el siguiente pixel de la altura " $h$ ", hasta recorrer toda la imagen.

> Se selecciona la imagen siguiente, repitiendo los pasos anteriores, hasta procesar todas las imágenes.

$>$ Se escriben todas las coordenadas en un archivo “.obj”, que es el objeto reconstruido en $3 \mathrm{D}$.

Transformación de coordenadas: Para llevar a cabo la reconstrucción se debe convertir un punto "P" de la imagen a uno en el plano $(\mathrm{x}, \mathrm{y}, \mathrm{z})$. Los parámetros marcados conocidos en la figura 1 son:

> $\mathrm{DC}=$ distancia desde la cámara al cen- tro de la plataforma.

$>\mathrm{DL}=$ distancia desde el láser al centro de la plataforma.

$>\alpha$ = ángulo formado entre el haz del láser y el centro de la cámara.

$>\beta=90^{\circ}-\alpha$

$>\mathrm{Xpx}=$ ancho total “w” en pixeles de la imagen (representado por la línea verde)

$>\mathrm{Opx}=$ punto cercano al centro de la imagen que será la distancia 0 para la reconstrucción.

$>\mathrm{Ppx}=$ distancia en pixeles desde $\mathrm{Opx}$ hasta el punto de interés que se reconstruirá en $3 \mathrm{D}$.

También se hace mención a "Zpx" que es la altura " $h$ " que no se encuentra dibujada en el diagrama por simplicidad.

La transformación de la coordenada " $\mathrm{z}$ " en el espacio 3D es la más directa y se corresponde con la coordenada " $h$ " de la imagen multiplicada por un factor de escala. Experimentalmente se obtuvo:

$$
z=\frac{h}{2} \quad 1
$$

Para obtener los valores de los puntos " $\mathrm{x}$ " $\mathrm{e}$ "y" del espacio 3D es necesario conocer la distancia "r" (radio del objeto) para un ángulo " $\phi$ " determinado.

Observando la figura 1 se comprueba que:

$$
r=\frac{P p x}{\operatorname{sen}(\beta)} \quad 2
$$


y luego

$$
\begin{aligned}
& x=r \cdot \cos (\alpha) \\
& y=r \cdot \operatorname{sen}(\alpha)
\end{aligned}
$$

Completando de esta forma, las tres coordenadas en el espacio 3D.

Esta matemática es la manera más simple de llevar a cabo la reconstrucción, pero la misma no contempla correcciones debido a la deformación introducida por la cámara ni por la perspectiva de la foto obtenida.

Post-procesamiento: A la salida del programa anterior se obtiene una nube de puntos que, para poder enviarse a una impresora 3D, se debe convertir en un objeto sólido. En el prototipo presentado en este trabajo, este paso se realiza en forma manual utilizando el programa de uso libre MeshLab (link de internet MeshLab) que contiene los filtros necesarios para llevar a cabo una limpieza y reconstrucción del objeto. El

procedimiento consiste en:

> Seleccionar y eliminar los puntos que no correspondan al objeto deseado, ya sea por errores en la reconstrucción o porque escapan al tamaño del objeto.

$>$ Obtener un subconjunto de puntos que mejor represente al objeto: esto se logra desde el menú "Sampling -> Poissondisk Sampling" y tomando 150000 "sample points".

> Preparar la nueva nube de puntos para reconstruir la malla sólida: se utiliza la función "Filters -> Normals, Curvatures and Orientation -> Compute normals for point sets", eligiendo un valor superior a 16 para "Number of Neighbors".

$>$ Luego se construye el objeto solido mediante "Filters $\rightarrow$ Points $\rightarrow$ Surface Reconstruction Poisson".

Finalmente se exporta el objeto como archivo STL (3D Systems 1989), lo que ya permite recomponerlo con una impresora $3 \mathrm{D}$.

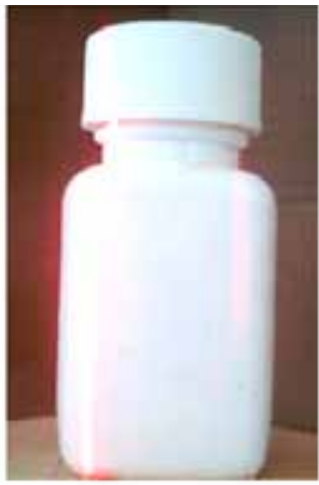

1

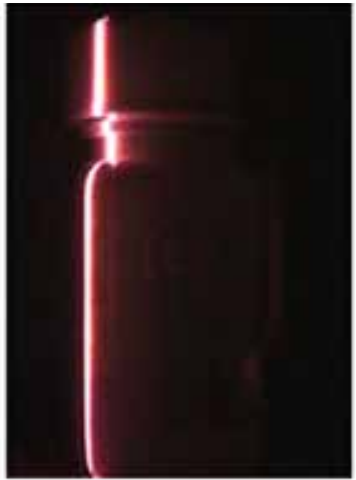

2

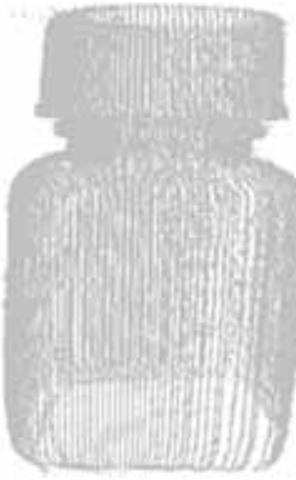

3

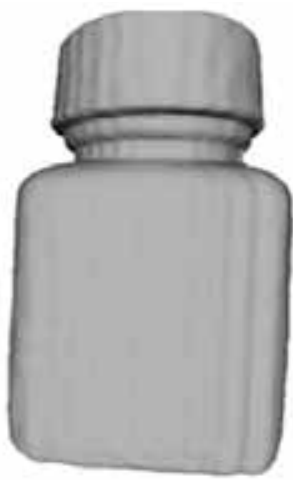

4

Figura 4.- Progresión del proceso completo. 1) Objeto real a escanear. 2) Imagen obtenida para un paso. 3) Nube de puntos representativa del objeto. 4) Objeto sólido listo para imprimir. 


\section{RESULTADOS}

Finalizadas las etapas anteriores y ya con el archivo en formato STL generado, se está en condiciones de imprimir el objeto con una impresora 3D. La figura 5 muestra, como ejemplo de aplicación del prototipo de escáner 3D, una botella plástica y su reconstrucción impresa en 3D por el método de extrusión utilizando la metodología explicada. La simple comparación de imágenes permite observar la distorsión angular, especialmente visible en la región de la tapa, debido a la falta de correcciones debido a la deformación introducida por la cámara ni por la perspectiva de la foto obtenida tal como se mencionó anteriormente.

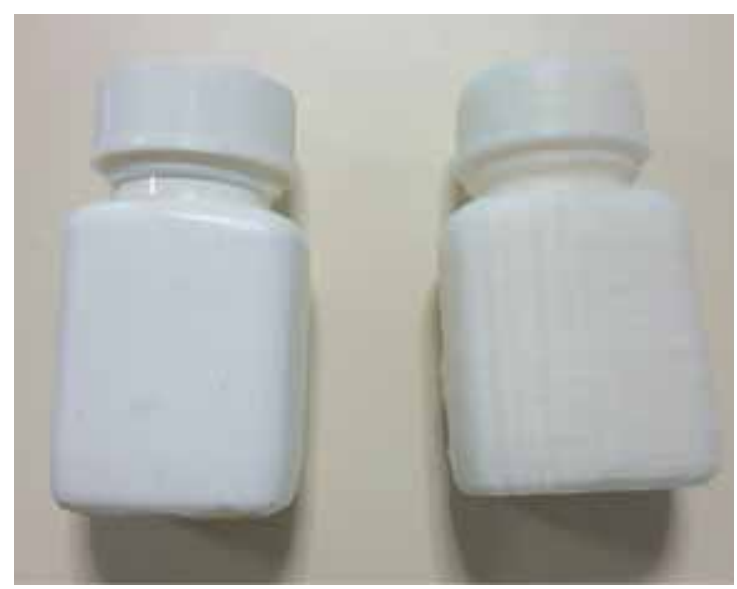

Figura 5.- Fotografía del objeto original (izquierda) y reconstruido (derecha)

Actualmente se está trabajando para incorporar en el algoritmo de tratamiento de imágenes correcciones a estos efectos para evitar deformaciones de las copias impresas en $3 \mathrm{D}$.

\section{CONCLUSIONES}

El prototipo del escáner desarrollado y presentado en este trabajo permite la adquisición y posterior reproducción de objetos de tamaño reducido con una precisión limi- tada por los componentes constructivos de esta primera etapa del proyecto.

Utilizando la misma metodología y reemplazando algunos de estos componentes se puede llegar sin inconvenientes a la precisión que requiera una determinada aplicación.

Este primer prototipo permitió la comprensión y desarrollo de herramientas de programación para el manejo de imágenes 3D. Asimismo, puso de manifiesto los puntos débiles de la mecánica y la electrónica asociada y la manera de salvarlos en una próxima versión. Entre ellas podemos nombrar, mejorar la precisión del motor paso a paso, trabajar con un controlador del motor (driver) apropiado al motor empleado, mejorar resolución de la cámara empleada, replantear la fuente de alimentación para que satisfaga las prestaciones necesarias, implementar una carcasa que permita la obtención de imágenes en oscuridad, entre otros.

\section{AGRADECIMIENTOS}

Los autores desean agradecer a la Secretaría General de Ciencia y Técnica por el financiamiento concedido en la marco del proyecto F024-2014. 


\section{BIBLIOGRAFIA:}

Malik, A. S. (Ed.). "Depth Map and 3D Imaging Applications: Algorithms and Technologies: Algorithms and Technologies". IGI Global. 2011.

Teutsch C. "Model-based Analysis and Evaluation of Point Sets from Optical 3D Laser Scanners”. 2007. Volume 1. Shaker Verlag, ISBN: 978-3-8322-6775-9.

Piegl, L., \& Tiller, W. "Curve and surface constructions using rational B-splines. ComputerAided Design”. 1987. Vol. 19(9), pp. 485498.

Millman J. y Halkias C. "Integrated Electronics: Analog and Digital Circuits and Systems". 1976. pp. 583-584. McGraw-Hill.

Microchip-hoja técnica.http://ww1.microchip. com/downloads/en/DeviceDoc/30487D. pdf

Sharp - hoja técnica. http://www.rcscomponents.kiev.ua/datasheets/pc817s.pdf

Kicad. http://www.kicad-pcb.org/display/ $\mathrm{KICAD} / \mathrm{KiCad}+\mathrm{EDA}+$ Software+Suite

Marder F., Toranzos V. , Aquino C. , Lombardero O., Marder V. "Módulo para ensayo de motores paso a paso". Ingeniería de Control. 2008. Vol. 88, pp.28-31.

Python. https://www.python.org/

OpenCV.http://opencv.org/

Calibración de la Cámara. https:// opencv-python-tutroals.readthedocs.io/en/ latest/py_tutorials/py_calib3d/py_calibration/py_calibration.html\#calibration

Formato OBJ http://www.cs.utah. edu/ boulos/cs3505/obj_spec.pdf

MeshLab. http://meshlab.sourceforge. net/

3D Systems, 1989, StereoLithography Interface Specification. 